\title{
Development of an efficient, highly uniform Sun Simulator for optical space instrument calibration
}

Simon Strotmann, Volker Kirschner, Dana Tomuta, Niels Dijkhuizen, Ralph Snel

Simon Strotmann, Volker Kirschner, Dana Tomuta, Niels Dijkhuizen, Ralph Snel, "Development of an efficient, highly uniform Sun Simulator for optical space instrument calibration," Proc. SPIE 11852, International Conference on Space Optics - ICSO 2020, 118526L (11 June 2021); doi: $10.1117 / 12.2600304$

SPIE Event: International Conference on Space Optics - ICSO 2021, 2021, Online Only 


\section{International Conference on Space Optics-ICSO 2020}

Virtual Conference

30 March-2 April 2021

Edited by Bruno Cugny, Zoran Sodnik, and Nikos Karafolas
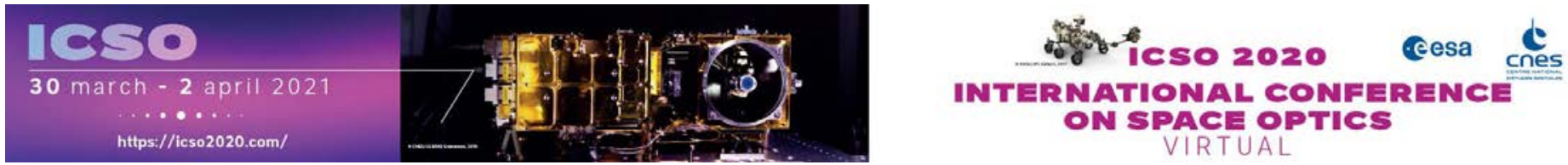

\section{Development of an efficient, highly uniform Sun Simulator for optical space instrument calibration}

\section{Cesa isoporecestings denes}




\title{
Development of an efficient, highly uniform Sun Simulator for optical space instrument calibration
}

\author{
Simon Strotmann ${ }^{\mathrm{a}}$, Volker Kirschner ${ }^{\mathrm{a}}$, Dana Tomuta ${ }^{\mathrm{a}}$, Niels Dijkhuizen ${ }^{\mathrm{b}}$, and Ralph Snel $^{\mathrm{b}}$ \\ aEuropean Space Agency (ESA), ESTEC, Keplerlaan 1, NL-2200 AG Noordwijk, The \\ Netherlands \\ bNetherlands Organisation for Applied Scientific Research (TNO), Stieltjesweg 1, 2628 CK, \\ Delft, the Netherlands
}

\begin{abstract}
In space Earth Observation related projects, Sun Simulators are regular used for on-ground calibrations of the flight instruments. Instrument radiometric calibration is established by illuminating the on-board diffuser(s) via the Sun port in irradiance configuration and an external OGSE calibrated diffuser for the radiance configuration via the Earth Port. Usually, Sun Simulator designs are complex containing multiple coated mirrors, and its implementation requires large volume and mass.

The paper will present an elegant and innovative concept of building such a Sun Simulator using minimum elements. The new concept consists of three major units: the illumination unit, the diffuser unit and the collimator unit. All units have been purchased, mounted on an optical table and aligned. Finally, preliminary performance tests of the Sun Simulator Breadboard are presented. The Breadboard proves the concept and also allows scaling to different instrument needs.
\end{abstract}

Keywords: Earth Observation, Sun Simulator, Optical Space Instrument Calibration

Further author information: (Send correspondence to Dana Tomuta)

Volker Kirschner: volker.kirschner@esa.int

Simon Strotmann: simon.strotmann@esa.int

Dana Tomuta.: dtomuta@cosmos.esa.int

Niels Dijkhuizen: niels.dijkhuizen@tno.nl

Ralph Snel: ralph.snel@tno.nl 


\section{INTRODUCTION}

All optical instruments for Earth Observation need to be calibrated on-ground in support to the in flight calibration. One element of this calibration process is the characterization of the calibration unit of the instrument, typically an internal diffuser generating a known radiance to the instrument in orbit when illuminated by the Sun. This characterization is performed by first illuminating the internal diffuser via the Sun calibration port by a source resembling the Sun and then repeating this process via the observation port using an accurately characterized external diffuser illuminated by the same source.

The source resembling the Sun is typically called Sun Simulator and has to have the same geometric illumination parameters as the Sun with high angular and spatial uniformity to avoid errors in the on ground calibration process. In addition, the Sun Simulator needs to ensure a spectro-radiometric output that is compatible with the radiometric sensitivity of the instrument. Usually, Sun Simulator designs are complex multi-mirror systems combined with illumination from an integrating sphere resulting in implementations with large volume and mass that are challenging regarding the handling during the calibration campaign. This paper will present the design and breadboard implementation of a Sun Simulator with a minimum number of components that results in a compact design yet meeting the needs for an accurate instrument calibration.

\section{DESIGN REQUIREMENTS}

Three requirements are driving the design of the Sun Simulator: the spatial uniformity of the simulated Sun, its angular uniformity and the irradiance that the Sun Simulator has to provide at its exit plane.

The review of requirements for a number of Sun Simulators used with different instruments (FLEX, Sentinel-4 and Sentinel-5) resulted in the following summary:

The illumination homogeneity of the Sun Simulator shall be better than $\pm 1.0 \%$ over the central area of the exit pupil, where the diameter of the central area varies per instrument: $150 \mathrm{~mm}$ diameter for Sentinel-4, $60 \mathrm{~mm}$ for Sentinel-5 and 100mm diameter for FLEX. The Sun Simulator exit pupil diameter is specified to be larger than $300 \mathrm{~mm}$ for Sentinel-4, $130 \mathrm{~mm}$ for Sentinel-5 and $200 \mathrm{~mm}$ for FLEX. The requirement for illumination uniformity of the Sun Simulator outside the central area of the exit pupil is usually more relaxed but could be still quite demanding as for Sentinel-5: better than $\pm 5.0 \%$ with respect to the central area.

The required illumination uniformity shall be within same value independent of the wavelength. The stability of the uniformity at the exit pupil shall be better than $\pm 0.5 \%$ within the central area of the Sun Simulator over a period of time that is usually representative for the duration of the planned measurement and is considering also the integration times that will be used.

Also, as part of Sun Simulator requirements, the beam divergence is specified to secure the angular diameter of the Sun (about $0.5^{\circ}$ ). However, the irradiance at the exit plane of the Sun Simulator (radiant exitance) is clearly specified and varies with the instrument needs. The following examples are expressed in [photons $\mathrm{cm}^{-2} \mathrm{~nm}^{-1} \mathrm{~s}^{-1}$ ]: for S4 for UV/VIS up to $\sim 4 \cdot 10^{14}$ and NIR $\sim 6 \cdot 10^{14}$ and S5 for UV/VIS max. $\sim 2 \cdot 10^{15}$ and $1.6 \cdot 10^{16}$ for NIR. The requirements of Sentinel-5 were taken as reference for the Sun Simulator presented here.

\section{SUN SIMULATOR DESIGN}

The idea of the proposed concept is based on the fact that a lambertian volume diffuser will provide a uniform radiance when illuminated. In addition, any illumination of the diffuser will cause multiple scattering events within the volume diffuser and will, therefore, also provide a spatially uniform illumination source. This source will have to be combined with a suitable collimator to generate a virtual image of the Sun.

One of the simplest imaging system consists of a parabolic mirror with the diffuser placed in its focus. As an obscuration is not allowed, the parabolic mirror will have to be used in an off-axis configuration. Nevertheless, the mirror should remain as close as possible to its axis of symmetry to firstly limit any polarization of the collimated output and secondly, limit the non-uniformity of the output due to the change of the mirror curvature with the off-axis distance.

The actual lay-out and sizing of the breadboard presented in this paper is shown in Figure 1. With a focal length of the parabolic mirror of $1000 \mathrm{~mm}$ and an average off-axis distance of $153 \mathrm{~mm}$ (the distance between the 


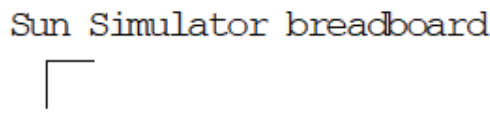

$449.23,87$
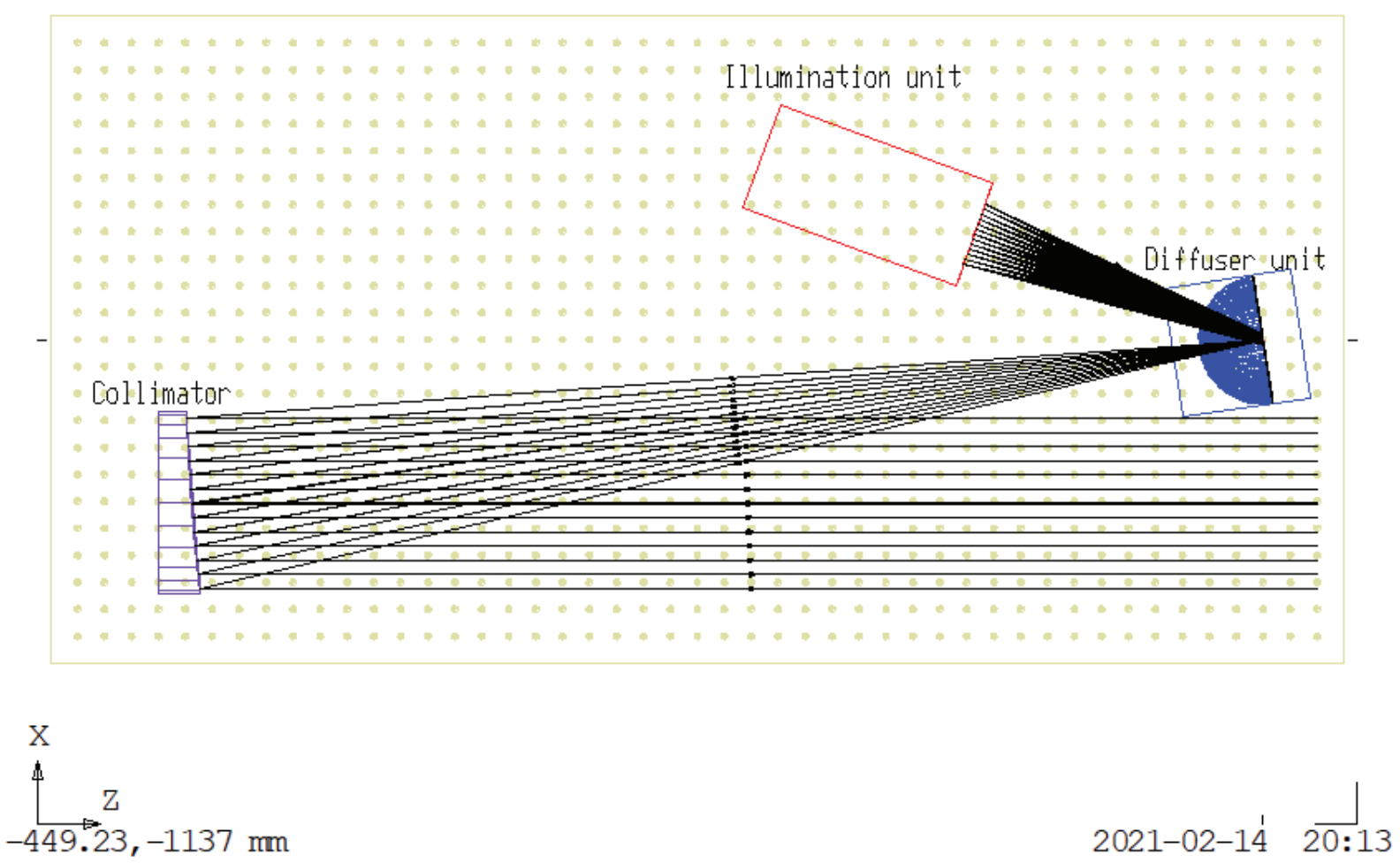

Figure 1. Optical lay-out of the Sun Simulator

axis of the parabola and the centre of the off-axis mirror), the spatial non-uniformity is limited to $0.5 \%$ across the mirror. This has been confirmed by an analysis in ASAP. The remainder of the uniformity budget is allocated to a deviation from a perfect lambertian scatter behavior of the diffuser, a possible coating non-uniformity of the mirror and residual straylight in the set-up. The diffuser diameter is chosen such that it represents the solar diameter of $0.54^{\circ}$.

The radiant exitance will have to be guaranteed by a sufficiently powerful source. A spherical mirror centered around the diffuser is introduced in order to reduce the light losses induced by the diffuser.

\subsection{Baffle Design}

In order to have a very good illumination uniformity as described above through the requirements of a Sun Simulator, baffles are foreseen to reduce the amount of straylight generated within the present breadboard. The baffles have to be placed at strategic positions in the system to suppress the light that propagates via undesired paths and influences the uniformity of the Sun Simulator exit beam.

The positions of the baffles where determined using ray tracing software (Zemax) in conjunction with a CAD model of the system. The baffle shapes and positions where determined by simulating the system in a reversed order, see Figure 2.

A source was simulated at the exit pupil of the system from which rays where traced backwards through the system. The ray trace confirmed that rays would either propagate via the nominal path or be blocked by the 


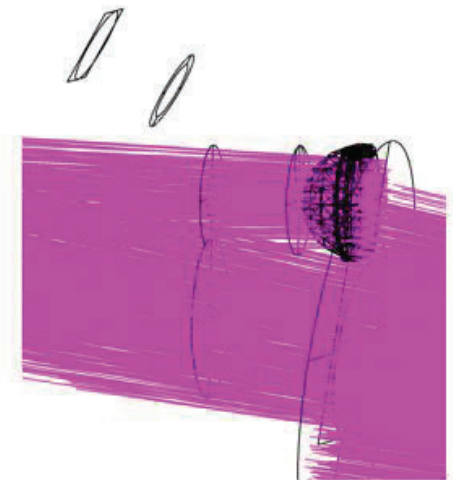

1. Simulate source at exit pupil

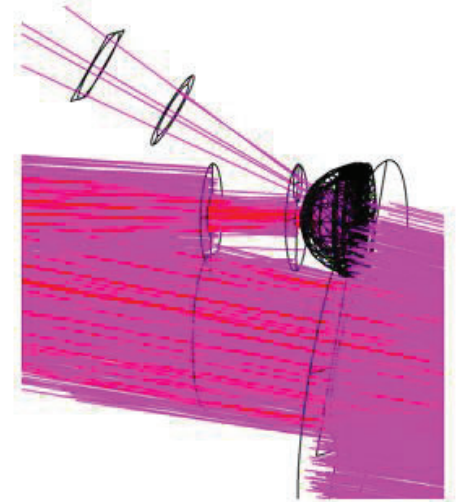

2. Block simulated source

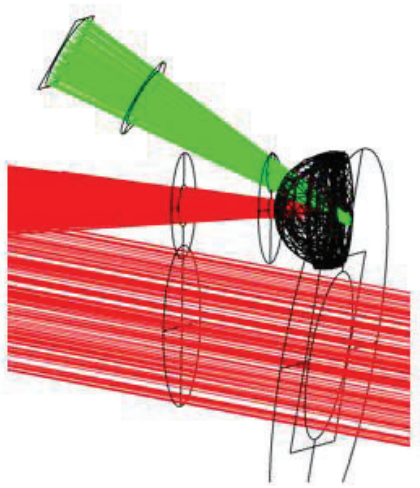

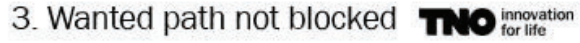

Figure 2. Zemax simulation of the baffle design provided by TNO.

baffle structures. This approach is very efficient in finding the correct baffle positions and their shapes, however it is less effective in predicting the straylight performance of the system quantitatively.

Tests of the Sun Simulator uniformity will finally confirm if the straylight suppression provided by the baffles is sufficient. If required, adaptations to the baffles can be made readily.

\section{HARDWARE IMPLEMENTATION}

The Sun Simulator breadboard comprises three main units: The illumination unit, the diffuser unit and the collimator unit. These three units are presented in this section and can be seen in Figure 3 showing a picture of the hardware implementation.

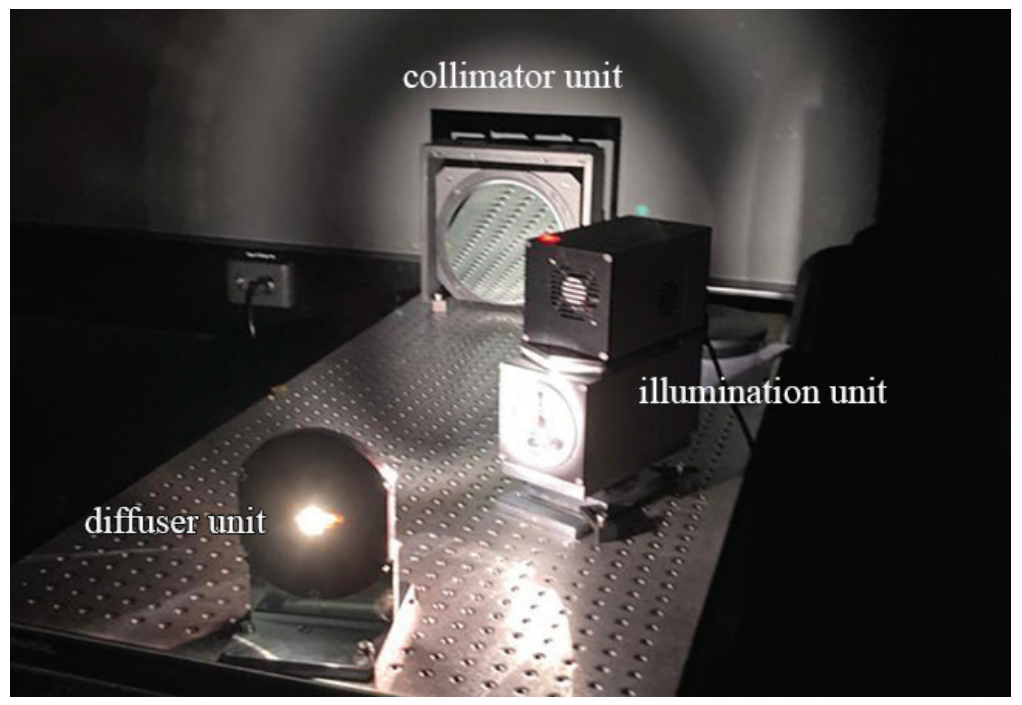

Figure 3. Picture of the hardware implementation showing the three main units: The illumination unit, the diffuser unit containing the diffuser and the collimator unit mounted on the base plate. 


\section{Illumination Unit}

In order to simulate the Sun characteristics as best as possible with respect to spectral range we selected a Xenon arc lamp as source. As the design of a lamp housing is not trivial for arc lamps we chose a solution that already incorporates lamp, collector and power supply in a convenient housing from Horiba - see Figure 4. A Horiba Xenon lamp of $150 \mathrm{~W}$ would be appropriate for the required exitance, however those are no longer available. The $75 \mathrm{~W}$ Xenon arc lamp (Horiba) was eventually selected for this Sun Simulator breadboard to demonstrate that the concept of this Breadboard design works and is compliant with the uniformity requirement although the radiant exitance might not be sufficient. Super-quiet higher power lamps by Hamamatsu could be a better solution with regard to the output stability but were not further explored.
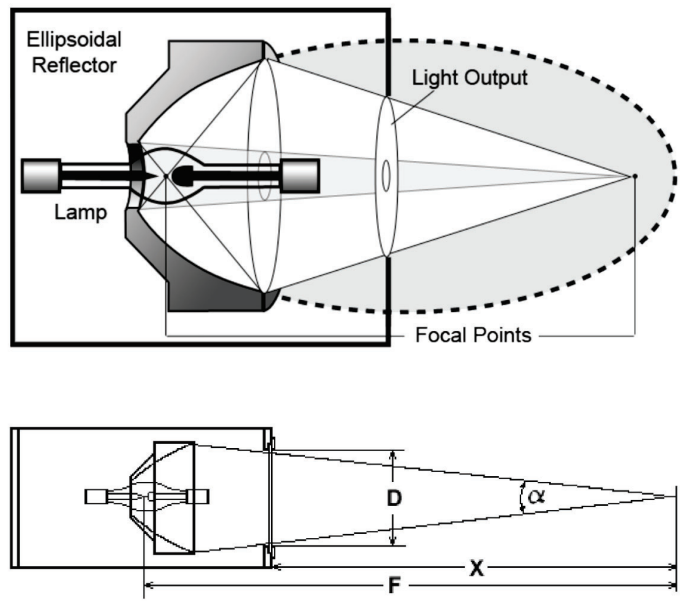

Figure 4. Dimensions of the light source. F: Focal length $(379 \mathrm{~mm})$, X: focal point from housing $(284.35 \mathrm{~mm}), \alpha$ : beam angle $\left(14.5^{\circ}\right), \mathrm{D}$ : window diameter $(65 \mathrm{~mm}){ }^{1}$

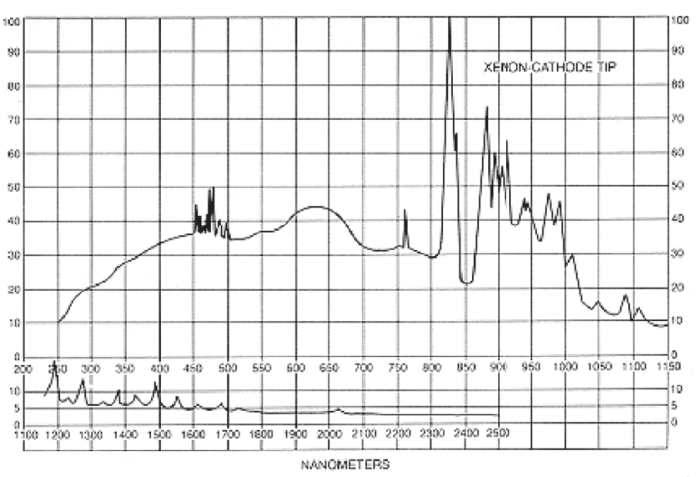

Figure 5. Xenon arc spectrum from Horiba specifications revealing the percentage of the total optical output power. ${ }^{2}$

The above mentioned Xenon lamps are lamps that emit a continuous spectrum ranging from UV to NIR and have a reasonable output also in the UV, see Figure 5. The $75 \mathrm{~W}$ Xenon lamp selected incorporates an ellipsoidal reflector with a focal length of $379 \mathrm{~mm}$ focusing the lamp arc into a spot with the size of $5.4 \mathrm{~mm}$ diameter at best focus. As the focusing is adjustable the spot size can be increased as needed. The housing of the lamp is mounted on a rotation stage to be able to position the focused illumination spot at the center of the diffuser. The housing of the lamp is mounted on a rotation plate to enable fine-adjustable and reproducible angles of incidence.

\section{Diffuser Unit}

The diffuser unit - called the dome in this paper - is a novelty in design and functionality although similar concept was previously considered for Calibration Unit in JWST-MIRI ${ }^{3,4}$ as on-board spatially and spectrally uniform blackbody illumination source.

The dome consists of a lambertian diffuser that will serve as secondary source for the collimator. It is covered by a concave spherical mirror with an opening angle of $90^{\circ}$ centered on the diffuser see Figure 6 . The dome needs two ports, an entrance port for the illumination beam and an exit port towards the collimator unit. For the present Sun Simulator breadboard, the dome was made from uncoated aluminum with a thickness of $10 \mathrm{~mm}$ whereas its internal surface was finished using a Single Point Diamond Turning technique to secure a high reflectivity. The diffuser is a volume diffuser. Beside the optical power/light generated by the illumination unit, also, the diffuser, will be exposed to a non-negligible thermal power generated. To avoid any potential diffuser degradation due to the thermal load, ceramic diffusers are preferred for the above design. For the current Sun 


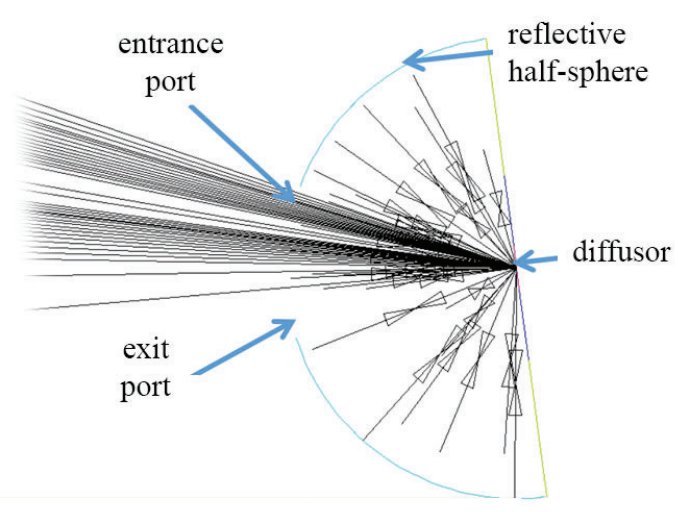

Figure 6. Top view of the dome with traced rays

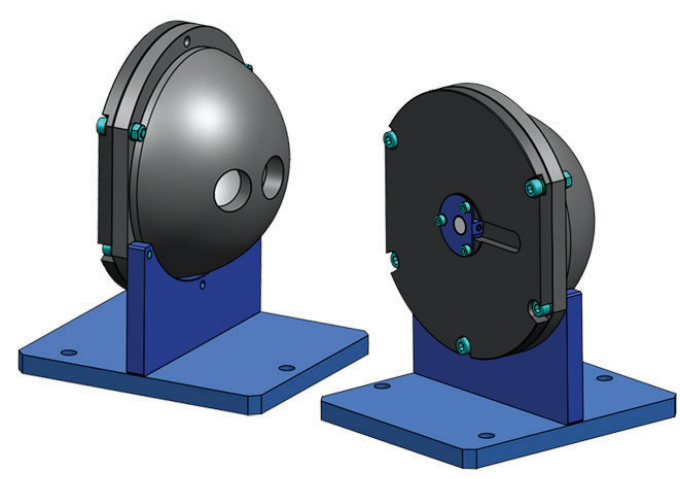

Figure 7. CAD rendering from the front (left) and back (right) view of the dome containing in the center of the dome the diffuser.

Simulator Breadboard, two diffuser options were found appropriate: HOD ${ }^{\complement}$ - High Purity Fused Silica Diffusor made by Heraeus and a similar type made by Opsira. Both of them have been used and the results are reported later in this paper.

To avoid unwanted residual reflections from within the dome, the aluminum back side around diffuser is blackened. The diffuser is held by grub screws and can be easily accessed and exchanged from outside the dome without changing dome position in respect to the illumination unit once aligned. A representation of the dome is given in Figure 7.

\section{Collimator Unit}

The collimator unit consists of an off-axis parabolic mirror and is placed such that its focus lies in the center of the diffuser. The off-axis mirror was manufactured based on custom specifications and features an off-axis distance between the parabolic axis and the geometrical center of the mirror of $153 \mathrm{~mm}$. Based on the requirements derived from the analytical model described in Section 2, the radius of curvature is $2000 \mathrm{~mm}$ with an optical clear diameter of $170 \mathrm{~mm}$ and a surface roughness below $2 \mathrm{~nm}$. The mirror is embedded in a mechanical structure with kinematic mountings to enable fine adjustments for alignment.

The alignment of all three units is not trivial and was done in several iterations to secure a well collimated beam. The off-axis mirror was aligned using a procedure involving two parallel laser beams. ${ }^{5}$

\section{PRELIMINARY TEST RESULTS}

Following the integration of the Sun Simulator the uniformity of the output beam was tested.

The test set-up consists of two S5973 Hamamatsu photo diodes connected to a Newport 2936-R power meter. One diode is placed in the proximity of the illumination unit and is used to monitor the flux of the lamp as a reference. The second diode is mounted on a XY linear translation stage that allows to scan the output beam over an area of $300 \mathrm{~mm} \times 300 \mathrm{~mm}$. This detection area is sufficiently large to validate the uniformity requirement of the sun simulator. The translation stage itself is placed as per design at a distance of $300 \mathrm{~cm}$ from the collimator unit. The measurement of the reference diode is taken into account such that fluctuations or drifts of the lamp output are not contributing in the processing of the uniformity scans.

The measurement scans are automated and controlled using LabView. They allow for setting the desired measurement area and its resolution and perform the read-out of the photo diodes. The pixels of the image are the result of a meander scan providing a measured power value of the second diode at each position, corrected by the measurement of the reference value of the first diode. In order to achieve a good signal-to-noise ratio and to prevent errors, other lights from the environment are switched off during measurements. 


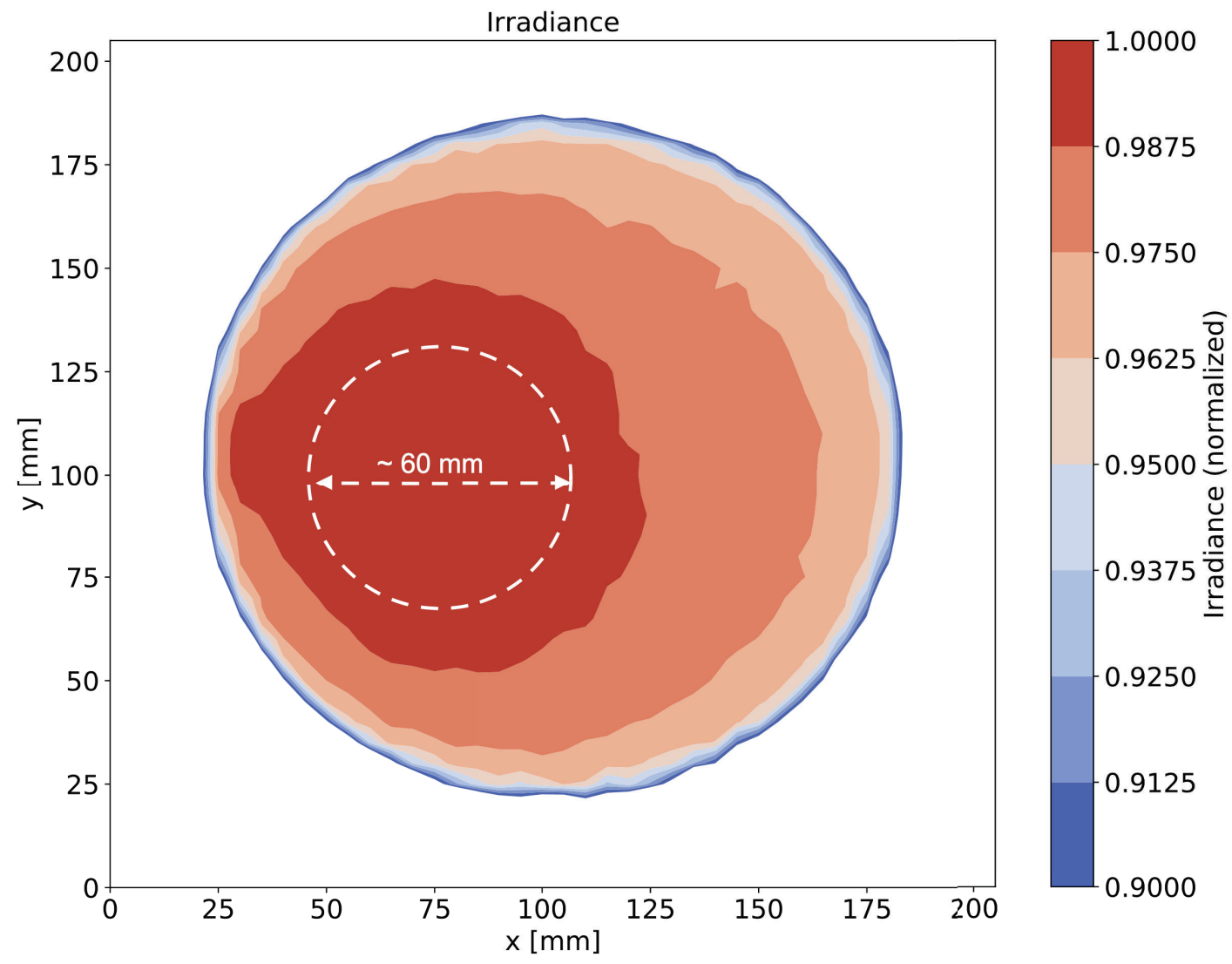

Figure 8. Normalized irradiance of the collimated beam at a distance of $\sim 300 \mathrm{~cm}$ from the mirror.

Figure 8 shows the results of such measurement scans and gives the beam uniformity presented as normalized intensity. Considering now the requirement for an illumination uniformity over $60 \mathrm{~mm}$ diameter (Sentinel-5), our first attempt reaches a value close to $\pm 0.5 \%$. in the region of interest. Figure 9 shows the vertical and horizontal intensity profile through the center position of the beam. From both Figure 8 and Figure 9 one can clearly notice a feature having an increased intensity placed on the left side of the collimated beam.
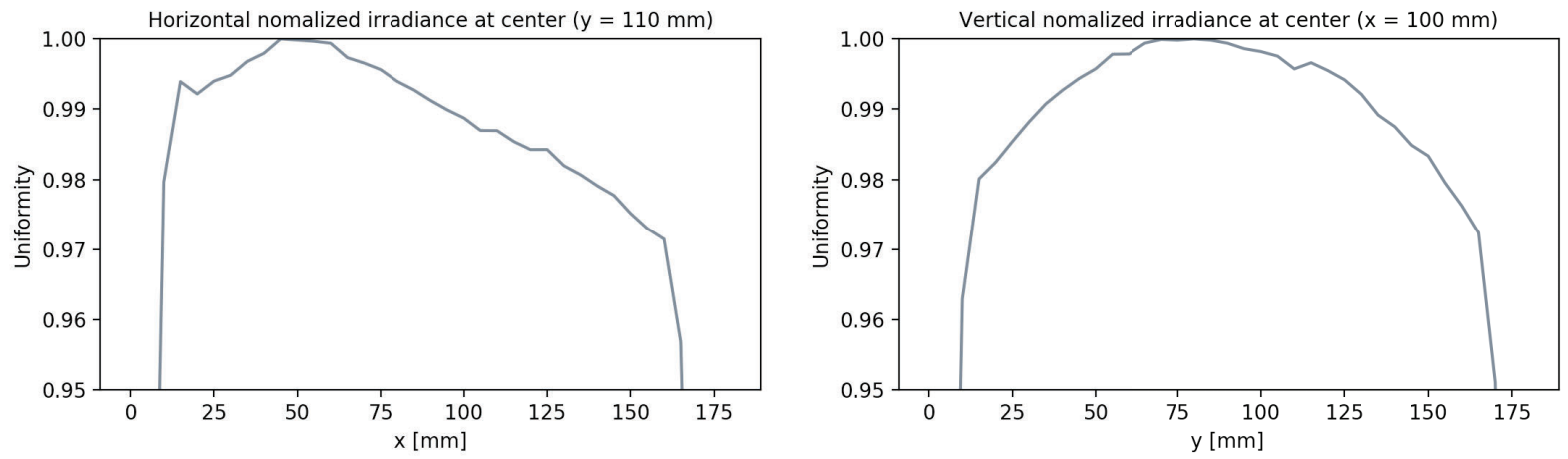

Figure 9. Profile of the normalized intensity through the center position of the collimated beam. 
Several checks regarding the diffuser material, alignment and the usage of a second mirror did neither affect the shape nor the position of this feature. Investigations for the cause of the remaining non-uniformity are still ongoing. Nevertheless, given the simple and elegant design of the breadboard, these results demonstrate already the potential of the presented approach of this Sun Simulator.

\section{FUTURE WORK}

As mentioned already in the results, the left side feature still needs to be understood and corrected to further improve the uniformity of the collimated outgoing beam. Because straylight effects could influence uniformity, a significant improvement is expected after the implementation of baffles at strategic positions in the system.

In addition to the spatial uniformity, the spectral uniformity will be measured. For this purpose convenient spectrometers were already identified and purchased. Two separate Hamamatsu spectrometers (C10083CA and C11118GA) will cover a large spectral range from $320 \mathrm{~nm}$ to $2550 \mathrm{~nm}$ with an intended redundant overlap region from $900 \mathrm{~nm}$ to $1000 \mathrm{~nm}$.

\section{REFERENCES}

[1] Horiba, "What makes the PowerArc a better Illuminator." Horiba https://static.horiba.com/ fileadmin/Horiba/Products/Scientific/Optical_Components_and_OEM/PowerArc/What_Makes_the_ PowerArc_a_Better_Illuminator.pdf (2020). (Accessed: 18 February 2021).

[2] Horiba, "Horiba Power Arc Additional Specifications." Horiba https://static.horiba.com/fileadmin/ Horiba/Products/Scientific/Optical_Components_and_OEM/PowerArc/PowerArc_Additional_ Specifications_01.pdf (2020). (Accessed: 18 February 2021).

[3] Wells, M., Pel, J.-W., Glasse, A., Wright, G. S., Aitink-Kroes, G., Azzollini, R., Beard, S., Brandl, B. R., Gallie, A., Geers, V. C., and et al., "The mid-infrared instrument for thejames webb space telescope, vi: The medium resolution spectrometer," Publications of the Astronomical Society of the Pacific 127, 646-664 (Jul 2015).

[4] Glasse, A., Lee, D., Parr-Burman, P., Hayton, D., and Mazy, E., "Onboard calibration sources for the midinfrared instrument (MIRI) on the James Webb space telescope," in [Space Telescopes and Instrumentation I: Optical, Infrared, and Millimeter], Mather, J. C., MacEwen, H. A., and de Graauw, M. W. M., eds., 6265, 1070 - 1076, International Society for Optics and Photonics, SPIE (2006).

[5] Lee, Y. H., "Alignment of an off-axis parabolic mirror with two parallel He-Ne laser beams," Optical Engineering 31, 2287-2292 (Nov. 1992). 